\title{
Medizin und Ökonomie
}

\author{
Regula Capaula, Thomas Brack ${ }^{\text {b }}$, Drahomir Aujesky
}

${ }^{a}$ Dr. med., Co-Präsidentin SGAIM; ${ }^{b}$ PD Dr. med., Präsident ICKS; ${ }^{c}$ Prof. Dr. med., Co-Präsident SGAIM

\author{
Die Schweizerische Gesellschaft für Allgemeine Innere Medizin (SGAIM) und der \\ Verein Internistischer Chef- und Kaderärzte (ICKS) nimmt Stellung zu den kürzlich \\ erfolgten Kündigungen/Freistellungen zweier verdienter Chefärzte der Spitäler Us- \\ ter und Bülach. Die Art und Weise der Kündigungen sorgen allgemein für Unver- \\ ständnis und Empörung.
}

Mit einer Mischung aus ungläubiger Überraschung, Ärger und Befremden registriert die SGAIM, die grösste medizinische Fachgesellschaft der Schweiz, die kürzlich erfolgte Entlassung von zwei kompetenten Chefärzten im Kanton Zürich. Zusammen mit der ICKS beobachten wir mit grosser Sorge die zunehmend ruppigere Gangart im Umgang mit verdienten und fachlich unbestrittenen Chefärzten medizinischer Kliniken durch eine vom Kerngeschäft abgekoppelte Spitaladministration. Die Identifikation mit Patienten und Betrieb ist bei Medizinern häufig stark ausgeprägt, weshalb eine Freistellung besonders tiefe Wunden hinterlässt. Die Ereignisse in Uster und Bülach machen betroffen und rufen nicht nur in medizinischen Kreisen Empörung hervor.

Die Aufgaben der Ärzte in einer Spitalleitung umfassen neben dem operativen Geschäft auch die Vertretung ihrer Kernaufgabe, nämlich dem medizinischen Wohlergehen der Patienten. Sowohl Esther Bächli als auch Nic Zerkiebel verfügen neben dem ärztlichen Diplom über einen akademischen Abschluss in Business Administration. Man kann ihnen nicht vorwerfen, dass sie in operativen und ökonomischen Aspekten der Betriebsführung unerfahren seien.

Betriebswirte vertreten die ökonomische Seite, welche durch politische Vorgaben, Qualitätsansprüche und die Digitalisierung zunehmend schwieriger im Lot zu halten ist. Der Spitalleitung steht meist ein Spitaldirektor (CEO) vor, der die Interessen der Spitalleitungsmitglieder abgleichen und vernünftige Kompromisse orchestrieren muss. Aktuell sind diese Herausforderungen im Gesundheitswesen wegen der Coronakrise zusätzlich gestiegen. Es überrascht entsprechend wenig, dass im Klima einer stark konkurrenzgeprägten Spitallandschaft wie Zürich die Entlassungen von zwei verdienten Chefärzten für Unmut sorgen. Kaum haben wir im Fall Uster Stellung bezogen, folgt das nächste Drama in Bülach. Das vereinbarte Stillschweigen aller Parteien über die eigentlichen Gründe der Differenzen lässt viel Raum für Spekulationen und verunmöglicht eine einleuch- tende Interpretation der Ereignisse. Äussern können wir uns allerdings über die Art und Weise des Vorgehens. Dieses lehnen wir kategorisch ab und geben Folgendes zu bedenken:

Es wird zunehmend anspruchsvoller, zwischen den Spitalleitungsmitgliedern einen Konsens zu erreichen. In solch schwierigen Situationen ist ein sachliches und überlegtes Vorgehen gefragt. Das von den CEOs der betroffenen Spitäler praktizierte Muster von Hire and Fire mit öffentlich zur Schau gestellter Freistellung ist abzulehnen. Das Bild eines unbescholtenen Chefarztes, der von zwei Security-Mitarbeitern in einem Überraschungscoup vom Arbeitsplatz aus dem Spital sekundiert wird, hinterlässt Ohnmacht, Wut und Misstrauen. In Bülach ist dies unter den Augen der Mitarbeitenden geschehen. Und hier wird es kompliziert:

Der CEO von Bülach, Rolf Gilgen, ist gleichzeitig Präsident der Vereinigung der Spitaldirektoren der Schweiz (SVS). Mit dieser Position geht die Erwartung einer speziellen Vorbildfunktion einher. Diese wurde in eklatanter Weise verletzt. Das unangemessene Vorgehen kann nicht kommentarlos hingenommen und akzeptiert werden. Die abschliessende Beurteilung, ob das Bild des in Bülach erzeugten Scherbenhaufens mit dieser Vorbildfunktion einhergeht, ist nicht unsere Aufgabe. Gefordert sind in dieser Situation der Verwaltungsrat und die Vereinigung der Spitaldirektoren. Wir sind der Meinung, dass solche Machtdemonstrationen von Spitaldirektionen gegen ärztliche Vertreter nur Verlierer hinterlassen und den immer grösser werdenden Graben zwischen Kerngeschäft und Administration weiter vertiefen. In dieser Hinsicht appellieren wir an alle Stakeholder, auch in schwierigen Situationen besonnen vorzugehen. Wir alle sind angehalten, unsere Vorbildfunktion helvetischen Gepflogenheiten und nicht Wild-West-Manieren anzugleichen. Wir stehen für einen partizipativen und kooperativen Führungsstil ein, welcher nach wie vor deutlich mehr Erfolg verspricht als Top-down-Entscheide. 